\title{
Visualizing Bond Types with Electron Density Models: How Informative is Electronegativity?
}

\author{
Heath D. Stotts and J. Conceicao* \\ Division of Natural Sciences \\ Northwestern Oklahoma State University \\ 709 Oklahoma Blvd. \\ Alva, Oklahoma 73717 USA
}

Received: March 2, 2006 Accepted: April 4, 2006

\begin{abstract}
Electron densities are used to visualize pure covalent, polar covalent and ionic bonds in binary compounds. The rationale for this study stems in part from the observations that within the same bond type, for example pure covalent, a variety of bond properties exist. Simple $\Delta E N$ predictions by Pauling do not adequately explain differences within the same bond type, nor determine covalent or ionic bonding. In this study, a series of electron density maps for binary compounds have been calculated to compare the characteristics of the maps to $\Delta \mathrm{EN}$ predictions.
\end{abstract}

\section{INTRODUCTION}

The concept of chemical bonding is by far one of the most useful, and at the same time one of the most difficult to understand in all of chemistry [1]. Understanding the structure and connectivity in bonds may allow us to better predict the properties of compounds and lead to the development of new materials, polymers, and other advanced technologies such as thin films, synthetic fibers and artificial blood. The importance of this concept was recognized by van Arkel who classified a bond as one of three types, these being: pure covalent, polar covalent, and ionic [2]. Specifically, a pure covalent bond exhibits equally shared electron density between the two bonding atoms with no net dipole moment. A polar covalent bond exhibits a shift in the bonding electron density and has a net dipole moment. At the furthermost extreme of the bonding spectrum ionic bonds are characterized by a lack of bonding electron density between the two bonding nuclei. In this case, the largest dipole moment is expected.

* The authors may be contacted through their e-mail addresses at Hdstotts@nwosu.edu and jjconceicao@nwosu.edu.

\begin{abstract}
The modern description of bond types is based on the relative electronegativity of the atoms involved in the bond. Bond type can be predicted using different models; the most prevalent one has been Pauling's difference of electronegativity $(\triangle \mathrm{EN})$ and is found to be accurate in most cases [3]. An exception to Pauling's $\Delta \mathrm{EN}$ is the Lil molecule which is predicted to be polar covalent, but experimentally it is ionic in character. An alternative method has been proposed by Sproul [4]. According to his model, a more complete description of bond type requires the use of an additional parameter, this being the average of the electronegativities, $1 / 2\left(\chi_{A}+\chi_{B}\right)$, rather than simply $\Delta \mathrm{EN}$. Both models though are still incomplete; for example in the pure covalent bonds of $\mathrm{H}_{2}$ or $\mathrm{Cl}_{2}$, differences still exist in bond energies. Bond typing based solely on electronegativity $\left(\Delta \mathrm{EN}\right.$ and $\left.1 / 2\left(\chi_{A}+\chi_{B}\right)\right)$ is thus inadequate. Ideally, bond type should be determined from electron density measurements. Over the past decade, advancements in experimental and theoretical methods allow accurate determination of electron densities [5-6] for small molecules and crystals [7-8]. With the advent of inexpensive computers and computational software, electron density
\end{abstract}


maps [9-11] are now used routinely and extensively to visualize orbitals, electrostatic potential, and atomic and molecular size [12]. In this paper we examine the bonding in various binary compounds using electron density models; showing that such maps may be better in determining bond type when compared to relative electronegativity maps like that of Pauling's $\triangle E N$.

\section{METHODS AND PROCEDURES}

The electron density maps of $\mathrm{H}_{2}, \mathrm{Cl}_{2}$, $\mathrm{Br}_{2}, \mathrm{FI}$, and Lil respectively exhibit isosurfaces at $0.08 \mathrm{au}$ [6] and calculated at the Hartree-Fock level using the 3-21G* basis set in the Spartan '04 program. 2-D electron density contour maps for $\mathrm{KCl}$ and $\mathrm{RbCl}$ were calculated at the same level of theory using the same basis sets in the HyperChem 7.5 Student's Edition [13]. Geometry optimizations were performed on all the compounds prior to the calculations of electron density.

\section{RESULTS AND DISCUSSION}

\section{a. Pure Covalent}

The calculated electron density maps for a series of molecules $\left(\mathrm{H}_{2}, \mathrm{Cl}_{2}\right.$ and $\mathrm{Br}_{2}$ ) with pure covalent bonds are shown in Figure 1. All of the molecules display a symmetric distribution of electron density between the bonding atoms. For $\mathrm{H}_{2}$ the greatest electron density is localized between the bonding atoms while the diatomic species in the halogen group show a distinct dip/waist, or lower quantity of electrons between the bonding atoms.

A pure covalent bond is one that is stated as having $\triangle E N$ of 0 ; although this is correct there are some discrepancies in this broad statement when referring to the different experimental bond energies of each molecule (Table 1). Our study shows that the bonding electron densities for $\mathrm{H}_{2}, \mathrm{Cl}_{2}$ and $\mathrm{Br}_{2}$ vary with increasing atom radii from $\mathrm{H}$ to $\mathrm{Br}$ reflecting different degrees of pure covalency. The lack of a dip/waist in the bonding region of $\mathrm{H}_{2}$, atomic radii of $37 \mathrm{pm}$ [14], in contrast to a well-defined waist in $\mathrm{Br}$ $\mathrm{Br}$, atomic radii of $114 \mathrm{pm}$ [7], is a consequence of the increased atomic radii of the latter. This is expected to place the bonding electrons further from the nuclei thus reducing the attraction. This diminishes electron densities along the bond axis leading to longer and weaker bonds [6]. Our electron density maps correlate to this trend as is evident in Figure 1. Table 1 is also provided to compare the experimental data of the molecules within the same $\Delta \mathrm{EN}$.

\section{b. Polar Covalent}

Electron density maps for two polar covalent molecules, $\mathrm{FI}$ and Lil, are shown in Figure 2. FI and Lil have the same $\Delta \mathrm{EN}$ of 1.5 and are classified simply as polar covalent bonds according to Pauling's predictions; the electron densities of these two compounds tell a very different story though in regards to their respective bonding natures. In Lil, the lack of electron density in the bonding region is representative of an ionic bonding situation, while the $\mathrm{FI}$ compound shows an asymmetric sharing of electrons between the bonding nuclei indicative of a polar covalent bond. Furthermore, the large differences in dipole moments and bond energy (Table 2) also support the electron density predictions of Figure 2. Indeed, concluding FI [4] as polar covalent compounds and Lil [11] as ionic.

To understand this correlation, one can invoke simple bonding ideas involving orbital interaction. The energy of the valence $2 p(\approx-15$ hartrees) [15] orbital of fluorine and the valence $5 p(\approx-20$ hartrees) orbital of iodine are comparable in magnitude, and thus are expected to interact favorably to form a covalent bond in FI. Since the atomic orbital of fluorine is lower in energy, the bonding molecular orbital containing the electron pair would be fluorine-like, resulting in a slight negative charge about the fluorine nucleus. Lil on the other hand is quite different. The energy of the $2 \mathrm{~s} \approx \approx-5$ hartrees) valence orbital of lithium and the $5 p(\approx-20$ hartrees) orbital of iodine differ substantially and thus covalent bond formation between these orbitals diminishes. The bonding molecular orbital would be more iodine-like and the electron pair would reside in a region much closer to iodine [1617]. In the case of Lil, the energy mismatch is large enough that the electron pair resides exclusively on iodine thereby resulting in the formation of an ionic bond. This justification is consistent with the observations in Figure 2. In Table 2 experimental data is also 


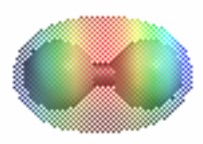

$\mathrm{H}_{2}$

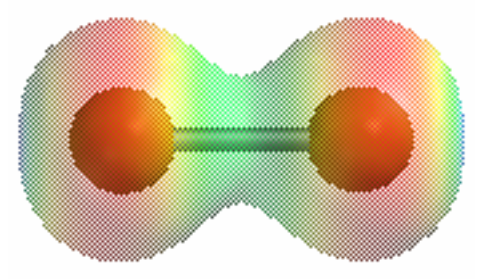

$\mathrm{Cl}_{2}$

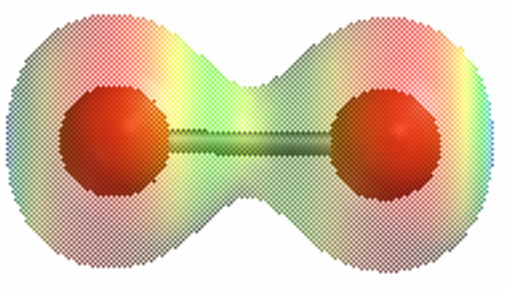

$\mathrm{Br}_{2}$

Figure 1. Pure Covalent: All models herein are scaled uniformly to represent their true relative sizes and were computed using the 3-21 $\mathrm{G}^{*}$ basis set at the Hartree-Fock level and at an isosurface electron density of 0.08 au. All models are represented by the same $\Delta \mathrm{EN}=0$. provided to better compare the characteristics of the two polar covalent molecules.

\section{c. Ionic Binary Compounds}

The 2-D contour electron density maps of two ionic compounds, $\mathrm{KCl}$ and $\mathrm{RbCl}$, are shown in Figure 3. In both instances, polarization of the electron clouds is observed. Both of these binary compounds have a $\Delta \mathrm{EN}$ of 2.2 and thus are ionic in nature. This simple classification does not explain the discrepancies in the experimental bond energies of $427 \mathrm{KJ} / \mathrm{mol}$ for $\mathrm{KCl}$ and $448 \mathrm{KJ} / \mathrm{mol}$ for $\mathrm{RbCl}$ as one would expect them to be identical given the apparent assumption that these bonds are the same. Furthermore bond lengths cannot be used to clarify this difference as it depends on the size of the ions.

To better understand this observation one has to consider the formation of covalent bonding in ionic compounds (covalency). The inter-nuclear electron cloud in $\mathrm{KCl}$ exhibits greater distortion representing a greater degree of covalent bonding while distortion to a lesser degree is observed in $\mathrm{RbCl}$ indicative of smaller covalent character. A greater degree of covalent character weakens the ionic bond in $\mathrm{KCl}$ thereby manifesting lower bond energy. In the opposition, $\mathrm{RbCl}$ has a lesser degree of covalent character resulting in higher bond energy.

\begin{tabular}{|c|c|c|c|c|c|c|}
\hline Molecule & $\Delta \mathrm{EN}$ & $\begin{array}{c}\text { Dipole } \\
\text { Moment } \\
(\text { Debye) }\end{array}$ & $\begin{array}{c}\text { Bond } \\
\text { Energy } \\
(\mathrm{kJ} / \mathrm{mol})\end{array}$ & $\begin{array}{c}\text { Atomic } \\
\text { Radii }\end{array}$ & $\begin{array}{c}\text { Bond } \\
\text { Length } \\
(\mathrm{pm})\end{array}$ & Density Model \\
\hline $\mathrm{H}_{2}$ & 0 & 0 & 436 & 37 & 74 & \\
\hline $\mathrm{Cl}_{2}$ & 0 & 0 & 243 & 99 & 199 & \\
\hline $\mathrm{Br}_{2}$ & 0 & 0 & 193 & 114 & 288 & \\
\hline
\end{tabular}

Table 1. Comparison of experimental data to density models for $\mathrm{H}_{2}, \mathrm{Cl}_{2}$, and $\mathrm{Br}_{2}$. 


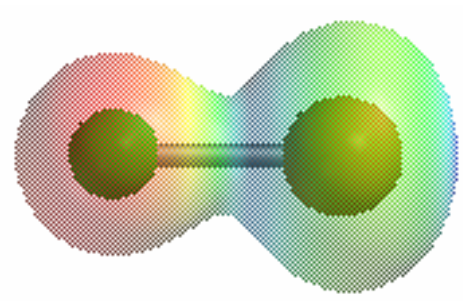

F-I

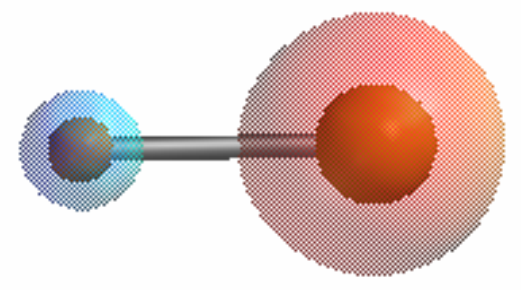

Li-I

Figure 2. Polar Covalent: Both models are scaled uniformly to represent true relative sizes, and were computed using the $3-21 \mathrm{G}^{*}$ basis set at the Hartree-Fock level and an isosurface electron density of $0.08 \mathrm{au}$. Both models represent the same $\Delta \mathrm{EN}=1.5$.

The presence of covalent bonding in ionic systems has previously been expressed in terms of the ionic potential [18]. The $\mathrm{K}^{+}$ion has a larger ionic potential as compared to the $\mathrm{Rb}^{+}$ion thereby exhibiting a larger degree of covalency. Table 3 (p. 14) shows an expanded view of the inter-nuclear region. The smaller distance " $\mathrm{C}$ " in $\mathrm{KCl}$ as opposed to "d" (larger) in $\mathrm{RbCl}$ provides a more quantitative measure of the degree of distortion in these two binary compounds. Fajans [19] proposed that cations of the same charge, but of decreasing size will polarize the anions to a greater extent. Our contour maps show a distortion of both electron clouds (cation and anion) in the inter-nuclear region rather than a one-sided polarization of the anion. These observations are not completely consistent with Fajans' rules, and a more extensive study of this discrepancy is underway. Results will appear in a future publication. Table 3 on page 14 also provides a summary of various experimental bond properties and the respective 2-D contour maps.

\section{CONCLUSION}

Electron density and electrostatic potential maps as well as 2-D contour maps were computed for a series of binary compounds. These maps provide better insight into the nature of each type of bond. These maps also include a more intuitive representation of bond strength in the pure covalent and ionic systems, and a more accurate method of bond typing in polar covalent systems.

\begin{tabular}{|c|c|c|c|c|}
\hline Molecule & $\Delta \mathrm{EN}$ & $\begin{array}{c}\text { Dipole Moment } \\
\text { (Debye) }\end{array}$ & $\begin{array}{c}\text { Bond } \\
\text { Energy } \\
(\mathrm{kJ} / \mathrm{mol})\end{array}$ & Density Model \\
\hline $\mathrm{F}-\mathrm{I}$ & 1.5 & 2.20 & 263 & \\
\hline $\mathrm{Li}-\mathrm{I}$ & 1.5 & 7.56 & 354 & \\
\hline
\end{tabular}

Table 2. Comparison of experimental data to density models for F-I and Li-I 

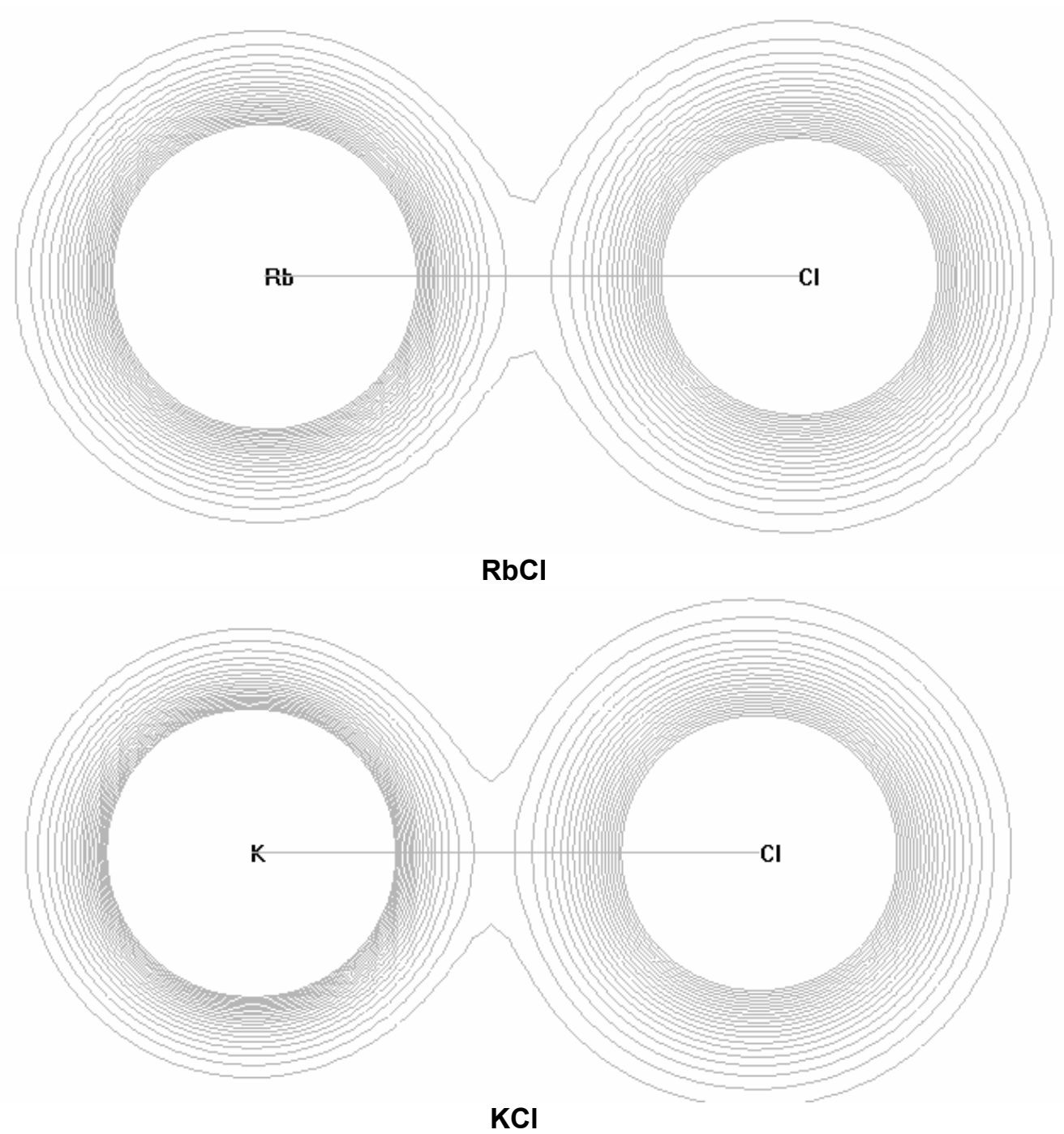

Figure 3. Ionic: Both 2-D contour models were calculated using a HF 3-21G* basis set at the Hartree-Fock level. Both models are sized uniformly to represent true relative size. Both models start with the outermost contour at an electron density of 0.02 au and have increments of 0.00875 au. Both models also represent a $\Delta \mathrm{EN}=2.2$.

\section{ACKNOWLEDGEMENT}

HDS and JC would like to thank Wavefunction Inc. for providing a 6-month evaluation copy of Spartan '04 for Windows.

\section{REFERENCES}

1. Brown, D. The Chemical Bond in Inorganic Chemistry-The Valance Bond Model (Oxford Science Publications. Oxford, 2002) p 3.
2. Arkel, A. E. V. Molecules and Crystals in Inorganic Chemistry (Interscience; New York, 1956).

3. Pauling, L. The Nature of the Chemical Bond, 3rd edn. (Cornell University Press: Ithaca, NY, 1967).

4. Sproul, G. J. Chem. Educ. 2001, 78, $387-390$.

5. Hehre, W.J. A Guide to Molecular Mechanics and Quantum Chemical Calculations; Wavefunction Inc.; Irvine, CA, 2003.

6. http://academic.reed.edu/chemistry/roco /Density/index.html 


\begin{tabular}{|c|c|c|c|c|c|c|}
\hline Molecule & $\Delta \mathrm{EN}$ & $\begin{array}{c}\text { Dipole } \\
\text { Moment } \\
\text { (Debye) }\end{array}$ & $\begin{array}{c}\text { Bond } \\
\text { Energy } \\
\text { (kJ/mol) }\end{array}$ & $\begin{array}{c}\text { Bond } \\
\text { Length } \\
\text { (pm) }\end{array}$ & $\begin{array}{c}\text { Ratio } \\
\text { "c" : "d" } \\
\text { (Unit } \\
\text { less) }\end{array}$ & Density Model \\
\hline $\mathrm{KCl}$ & 2.2 & 11.65 & 427 & 267 & 1 & \\
\hline $\mathrm{RbCl}$ & 2.2 & 11.93 & 448 & 279 & 1.2 & $<$ \\
\hline
\end{tabular}

Table 3. Comparison of experimental data to 2-D contour maps (inter-nuclear region) for $\mathrm{KCl}$ and $\mathrm{RbCl}$

7. Mori-Sanchez, P., Pendas, A. M., Luana, V. J. Am. Chem. Soc. 2002, 124, $14721-14723$.

8. Francl, M.M., Hout Jr., R.F., Hehre, W.J. J. Am. Chem. Soc. 1984, 106, 563 570.

9. Gilespie, R.J. J. Chem. Educ. 2001, 78, 1688 - 1691.

10. Matta, C.F., Gillespie, R.J. J. Chem. Educ. 2002, 79, $1141-1152$.

11. Rico, J.F., Lopez, R., Ema, I., Ramirez, G. J. Chem. Theory Comput. 2005, 1, $1083-1095$.

12. Shusterman, G.P., Shusterman, A.J. J. Chem. Educ. 1997, 74, 771 - 776.

13. HyperChem ${ }^{\circledR}$ Release 7 for Windows Reference Manual; Hypercube Inc.; 2002.

14. Silberberg, M.S. Chemistry: Molecular Nature of Matter and Change, 3rd edn. (McGraw-Hill Press; New York, NY, 2003).

15. Herman, F., Skillman, R. Atomic Structure Calculations (Prentice-Hall: Englewood Cliffs, N.J., 1963).

16. Coulson, C.A., McWeeny, R. Coulson's Valence, 3rd edn. (Oxford University Press; New York, NY, 1991).

17. Raff, L.M. Principles of Physical Chemistry, 1st edn. (Prentice-Hall, Inc.; Upper Saddle River, NJ, 2001).

18. Cartledge, G.H. J. Amer. Chem. Soc. 1930, 52, 3076.

19. Fajans, K. Naturwissenschaften 1923, $11,165$.

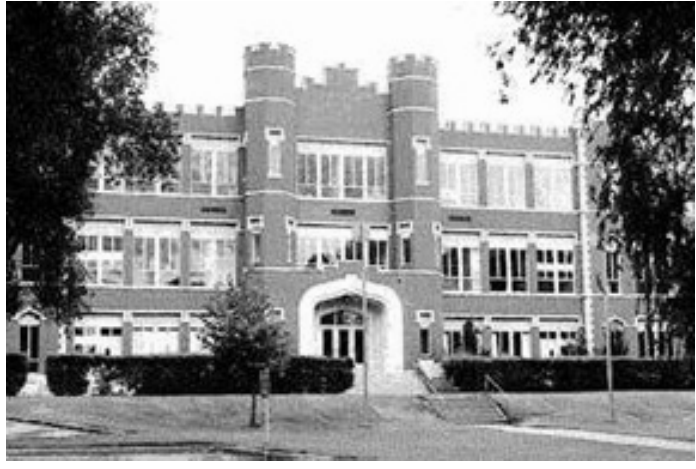

\section{NORTHWESTERN OKLAHOMA STATE UNIVERSITY}

Northwestern offers Bachelor of Arts and Bachelor of Science degrees in over 40 areas of study. A BS in Nursing degree is also offered. Some of the more popular fields of study include education, business administration, health and physical education, law enforcement, nursing, agriculture, psychology, pre-medicine, biology and mass communications. Study in several pre-professional fields is offered. At the graduate level, master's degrees in Education and Counseling Psychology are available. Information on the Graduate School and its program is available through the Graduate School Office by calling (580) 327-8410. A Master of Business Administration is available at the Alva, Enid and Woodward campuses through a cooperative effort with other universities.

http://www.nwalva.edu/ 


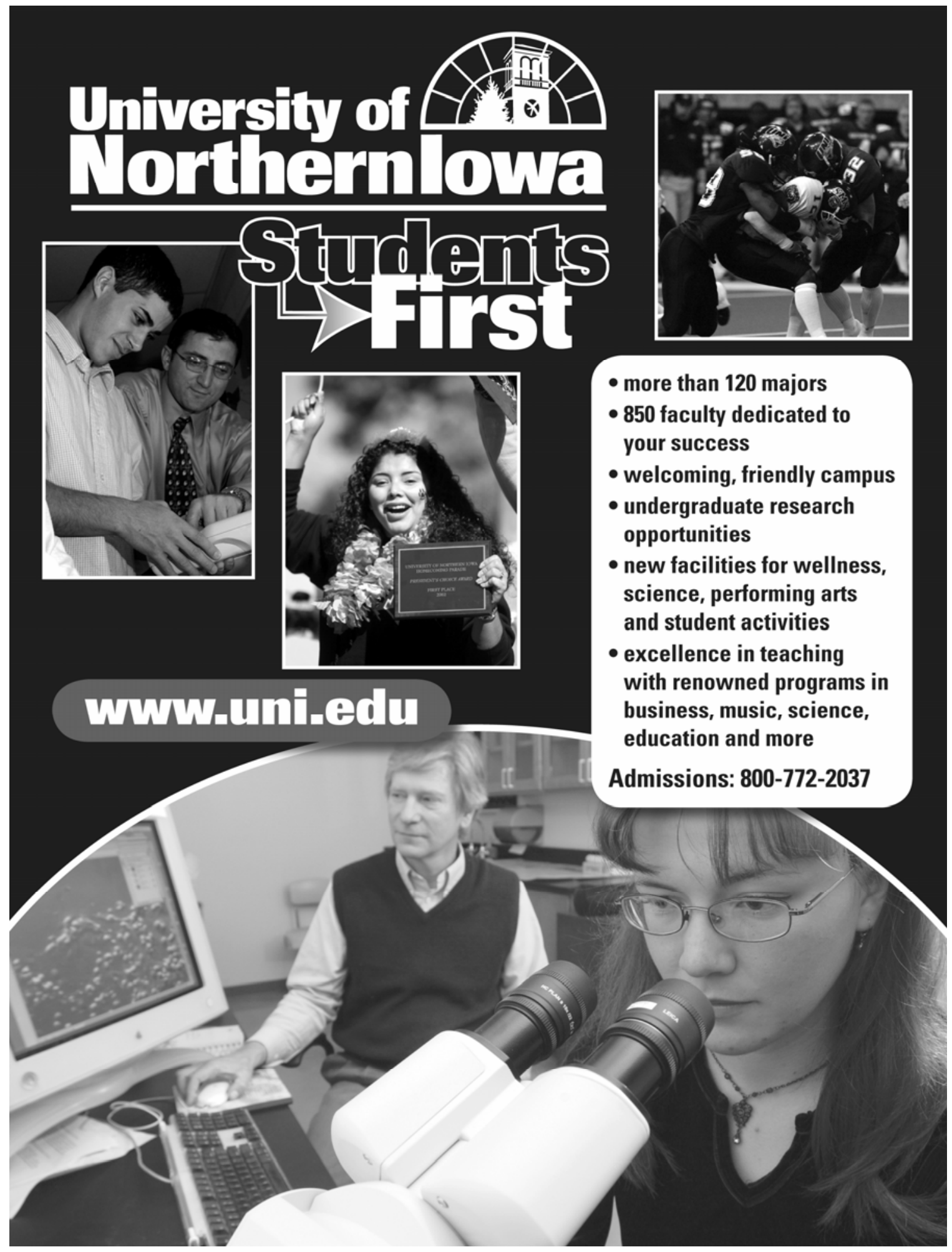






